\title{
Quantitative volumetric analysis of primary glioblastoma multiforme on MRI and 11C-methionine PET: initial study on five patients
}

\author{
Jingwang Zhao ${ }^{1}$, Zhijuan Chen², Li Cai, Shaoya Yin, Weidong Yang ${ }^{1}$, Zengguang Wang ${ }^{4}$ \\ ${ }^{1}$ Department of Neurosurgery, Tianjin Huanhu Hospital, No. 6 Jizhao Road, Jinnan District, Tianjin, China \\ ${ }^{2}$ Department of Neurosurgery, Tianjin Medical University General Hospital, Tianjin, China \\ ${ }^{3}$ Department of PET-CT Diagnostic, Tianjin Medical University General Hospital, No. 154 Anshan Road, Heping District, Tianjin, China \\ ${ }^{4}$ Tianjin Neurological Institute, No. 154 Anshan Road, Heping District, Tianjin, China
}

\begin{abstract}
To investigate the discrepancy between 11C-methionine (MET) positron emission tomography (PET) and MRI results in primary glioblastoma multiforme (GBM) through three-dimensional (3D) volumetric analysis, we retrospectively analysed patients with primary GBM who underwent preoperative 3D MRI and MET PET and were operated between June 2016 and January 2017. Tumour delineation and volumetric analysis were conducted using MRIcron software. Tumour volumes defined by MRI (VMRI) were manually drawn slice by slice in axial and sagittal or coronal images of enhanced $\mathrm{T} 1$ sequence, while metabolic tumour volumes were automatically segmented in MET PET (VMET) based on three (frontal, occipital and temporal) 3D reference volumes of interest (VOI). Discrepancies were evaluated in terms of both absolute volume and percentage on the combined images. MET PET contours contained and extended beyond MRI contours in all five patients; in a subset of cases, MET PET contours extended to the contralateral hemisphere. The discrepancy between MET uptake and MRI results was $27.67 \mathrm{~cm}^{3}\left(4.20-51.20 \mathrm{~cm}^{3}\right)$, i.e. approximately $39.0 \%(17.4-64.3 \%)$ of the metabolic tumour volume was located outside the volumes of the Gd-enhanced area. Metabolic tumour volume is substantially underestimated by $\mathrm{Gd}$-enhanced area in patients with primary GBM. Quantitative volumetric information derived from MET uptake is useful in defining tumour targets and designing individualised therapy strategies in primary GBM.
\end{abstract}

Key words: Volumetric analysis, glioblastoma multiforme, MRI, 11C-methionine

(Neurol Neurochir Pol 2019; 53 (3): 199-204)

\section{Introduction}

Glioma is the most common primary brain tumour of the central nervous system in adults (38\%). The World Health Organisation (WHO) has established criteria for classifying glioma into four histological grades according to the tumour's pathological morphology [1]. Glioblastoma multiforme (GBM), the highest grade glioma, is intensively infiltrative and has a diffuse border with the brain parenchyma. As a consequence, defining tumour boundaries for treatment is challenging. Magnetic resonance imaging (MRI) is the most widely used imaging modality and the standard reference for diagnosis, therapy planning and follow-up of GBM tumours. Inhomogeneous enhancement and necrosis characterise the majority of GBM lesions [2]. The standard MRI sequence for calculating tumour volume and planning the extent of surgery for high-grade gliomas is gadolinium (Gd) enhanced T1-weighted imaging [3]. However, MRI cannot precisely delineate tumour volume and is insufficient in helping to achieve total resection of glioblastoma, because $\mathrm{T} 2$-weighted imaging can incorporate both tumour tissue and perifocal oedema [4], and high-grade glioma cells are not limited to the Gd-enhanced volume in MRI T1 images [5, 6] and in fact can be located up to $30 \mathrm{~mm}$ beyond the Gd-enhanced area [7].

Address for correspondence: Weidong Yang, Department of Neurosurgery, Tianjin Huanhu Hospital, No. 6 Jizhao Road, Jinnan District, Tianjin, China, e-mail: yangweidongshine@yahoo.cn 
Positron emission tomography (PET) with metabolic tracers has played an important role in neurooncology $[8,9]$. Radiolabelled amino acids, such as ${ }^{11} \mathrm{C}$-methionine (MET) PET, reflect the extent of tumour boundaries in GBM more reliably than CT or MRI, because Gd-enhanced areas in MRI represent disruptions of the brain-blood barrier, while MET uptake correlates with tumour cell density or proliferative capability, thus more closely reflecting the properties of glioblastoma multiforme [10-13]. Complete resection of MET uptake areas has been shown to increase survival of high-grade glioma patients in previous studies $[14,15]$. Both qualitative and semiquantitative analysis of Gd-enhanced T1 and MET PET in GBM have revealed discrepancies between the two modalities [7, 16, 17], but these studies reported neither volumetric analysis of entire tumours nor volume information of any sort. To date, only a few studies have conducted volumetric calculation [18, 19], and no 3D volumetric analysis of discrepancies between MRI and MET PET has been performed focusing on primary GBM.

The current study therefore aimed to investigate differences in estimates of primary GBM tumour volume based on 3D MRI and MET PET.

\section{Materials and methods}

\section{Patients}

Firstly, we retrospectively reviewed the neurosurgery database and found a total of 461 brain tumour operations performed between June 2016 and January 2017. Secondly, patients were screened for pathological confirmation of GBM, with 31 patients thereby selected. Finally, patients were required to meet all of the following inclusion criteria: (1) they had no prior biopsy or treatment; (2) they had available data from preoperative $3 \mathrm{D}$ whole brain contrast-enhanced MRI and MET PET; and (3) the interval between these two examinations was less than seven days. The final study population consisted of five patients, whose clinical data is set out in Table 1 . The protocol of this study was approved by the Research Ethics Committee of the Hospital.

\section{Image acquisition}

MR images were obtained using a 3.0-Tesla MR scanner (Discovery MR750, General Electric, Milwaukee, WI, USA). Sagittal 3D T1-weighted images were acquired using a whole brain sequence with the following parameters: repetition time $(\mathrm{TR})=8.2 \mathrm{~ms}$; echo time $(\mathrm{TE})=3.2 \mathrm{~ms}$; inversion time $(\mathrm{TI})=450 \mathrm{~ms}$; flip angle $(\mathrm{FA})=12^{\circ}$; field of view $(\mathrm{FOV})$ $=256 \mathrm{~mm} \times 256 \mathrm{~mm}$; and matrix $=256 \times 256$; slice thickness $=1 \mathrm{~mm}$, no gap.

PET images were acquired with a dedicated PET/CT scanner (Discovery LS, GE Medical Systems, Milwaukee, WI, USA). Patients fasted for at least six hours before examination; a dose of 555-740 MBq (15-20 mCi) MET was injected intravenously within one minute. Static emission scanning was performed for a minimum of 20 minutes after MET injection in $3 \mathrm{D}$ mode $(\mathrm{FOV}=150 \mathrm{~mm} \times 150 \mathrm{~mm}$, slice thickness $=5.0 \mathrm{~mm}$, slice gap $4.5 \mathrm{~mm}$, matrix $=128 \times 128$ ). For attenuation correction, we acquired a non-contrast-enhanced, low-dose CT scan (slice thickness $=2.5 \mathrm{~mm}$, slice gap $=0$, matrix $=512 \times 512$ ). MET uptake in the tumour and in normal cortex was expressed as standard uptake value (SUV). The ratio of tumour SUV to normal SUV in contralateral gray matter (TNR) was calculated for each tumour by a nuclear medicine physician trained in brain PET. For surgical planning, the TNR was adjusted to 1.3 to define the tumour target.

\section{Image processing and volumetric analysis}

The MET PET and MRI images were first transferred from DICOM to NIfTI format for viewing and processing using MRIcron software (http://www.mricro.com; University of South Carolina, Columbia, SC, USA). Images were then coregistered using Statistical Parametric Mapping 12 software (http://www.fil.ion.ucl.ac.uk/spm/; Wellcome Department of Cognitive Neurology, London, UK).

Tumour delineation and volumetric analysis of MRI and MET PET images were performed in MRIcron. The tumour was defined as previously described $[18,20]$. Tumour volumes defined by MRI (VMRI) were manually drawn slice by slice in axial and sagittal or coronal images of the enhanced $\mathrm{T} 1$ sequence by two skilled radiologists. The metabolic tumour volume defined by MET uptake (VMET) was automatically segmented based on image thresholding. Firstly, three spherical regions of $10-\mathrm{mm}$ radius were drawn in the unaffected contralateral frontal, occipital and temporal parenchyma as reference VOI in order to calculate normal SUV. Secondly, VMET was automatically segmented by image thresholding $(\mathrm{TNR}=1.3)$ as defined above. For tumour volume comparisons between MET PET and the corresponding MRI, contours were extended to central areas without MET uptake related to necrosis. High uptake areas beyond the tumour were manually excluded in order to avoid inclusion of physiological MET uptake in normal tissues. Thirdly, in order to express tumour volume differences between the two modalities, volume of discrepancy-MET (dis-MET: VMET not included in VMRI) was evaluated (both in terms of absolute volume and percentage) using the 'Overlay Comparisons' and 'Descriptive' functions included in MRIcron.

\section{Statistical analysis}

Statistical analyses were performed using SPSS 19.0 software (SPSS Inc., Chicago, IL, USA). Descriptive statistics were expressed as median and range [median (range)]. Values of $p<0.05$ (two-tailed) were considered statistically significant.

\section{Results}

Patient characteristics and volumetric data for the whole group are summarised in Table 1. 
Table 1. Patient characteristics and volumetric analysis of MRI and MET PET

\begin{tabular}{lccccccc} 
No. Sex & Age & $\begin{array}{c}\text { Location } \\
\text { (side) }\end{array}$ & $\begin{array}{c}\text { VMRI } \\
\left(\mathbf{( c m}^{3}\right)\end{array}$ & $\begin{array}{c}\text { VMET } \\
\left(\mathbf{c m}^{\mathbf{3}}\right)\end{array}$ & $\begin{array}{c}\text { dis-MET } \\
\left(\mathbf{c m}^{\mathbf{3}} \mathbf{)}\right.\end{array}$ & $\begin{array}{c}\text { dis-MET } \\
(\%)\end{array}$ \\
\hline 1 & F & 63 & Frontal(R) & 53.81 & 93.31 & 45.15 & 48.4 \\
2 & M & 66 & Temporal(L) & 43.86 & 59.20 & 16.80 & 28.4 \\
3 & M & 71 & Occipital(L) & 38.48 & 57.43 & 20.98 & 36.5 \\
4 & F & 66 & Frontal(R) & 22.24 & 24.12 & 4.20 & 17.4 \\
5 & F & 65 & Frontal(L) & 28.92 & 79.63 & 51.20 & 64.3
\end{tabular}

F femal, GBM glioblastoma multiforme, L left, M male, No. patient number, R right

On MET PET, uptake in the metabolically active tumour was clearly differentiated from that of normal brain tissue for all five patients. Visual analysis of coincidence and discrepancy between MET high uptake volumes and Gd-enhanced MRI demonstrated that MET PET contours contained and extended beyond most MRI contours. Furthermore, MET PET volumes even extended along commissural fibres to the contralateral cerebral hemisphere (Figure 1). Moreover, the overall shape of MET high uptake volumes was asymmetrical and irregular.

In volumetric analysis, the discrepancy of MET uptake was $27.67 \mathrm{~cm}^{3}\left(4.20-51.20 \mathrm{~cm}^{3}\right)$, i.e. about $39.0 \%$ (17.4-64.3\%) of metabolically defined tumour volumes were located beyond the Gd-enhanced regions (Figure 2).

\section{Discussion}

Glioblastoma multiforme is known for its invasive and aggressive behaviour, and there is no distinct margin with the brain parenchyma [1]. Gd-enhanced T1-weighted MRI is the standard MRI sequence for tumour delineation and defining resection targets for high-grade gliomas [3]. Timothy et al. conducted the largest systematic review and the only quantitative meta-analysis to investigate associations between the extent of resection and overall progression-free survival in GBM. They concluded that compared to subtotal resection, gross total resection substantially improved overall and progression-free survival [21]. Although cytoreductive surgery is the cornerstone of therapy in GBM, no consensus exists regarding the optimal extent of tumour resection necessary to improve survival [11]. Most GBM still recurs even after removal of all contrast-enhanced volumes on T1-weighted MRI. Therefore techniques such as metabolic imaging are needed to improve resection rates and the safety of surgery.

A subset of studies has reported that MET uptake reflects proliferation potential and angiogenic capability in gliomas $[22,23]$. In these studies, MET uptake was compared to the Ki-67 labelling index and microvessel density non-stereotactically. To overcome this problem, Yoshiko et al. conducted a stereotactic comparison of MET PET images and the resulting histology; they confirmed that MET uptake correlates with tumour cell density rather than with microvessel density in glioma [10]. They concluded that MET uptake correlates with tumour cell density (proliferative capability). Moreover, Yoo et al. [15] reported that metabolic tumour volume on MET PET is a significant and independent prognostic factor for progression-free survival in high-grade glioma; they recommended volumetric analysis of MET for better prognostication. Singhal et al. [24] compared MET to ${ }^{18}$ F-fluorodeoxyglucose (FDG) and contrast enhancement on MRI, suggesting that MET PET can predict prognosis in gliomas more accurately than FDG PET and MRI. In summary, MET is more accurate in delineating the tumour and defining the extent of resection targets.

Given the different physiological bases of Gd-enhanced MRI and MET uptake, discrepancies between MRI and
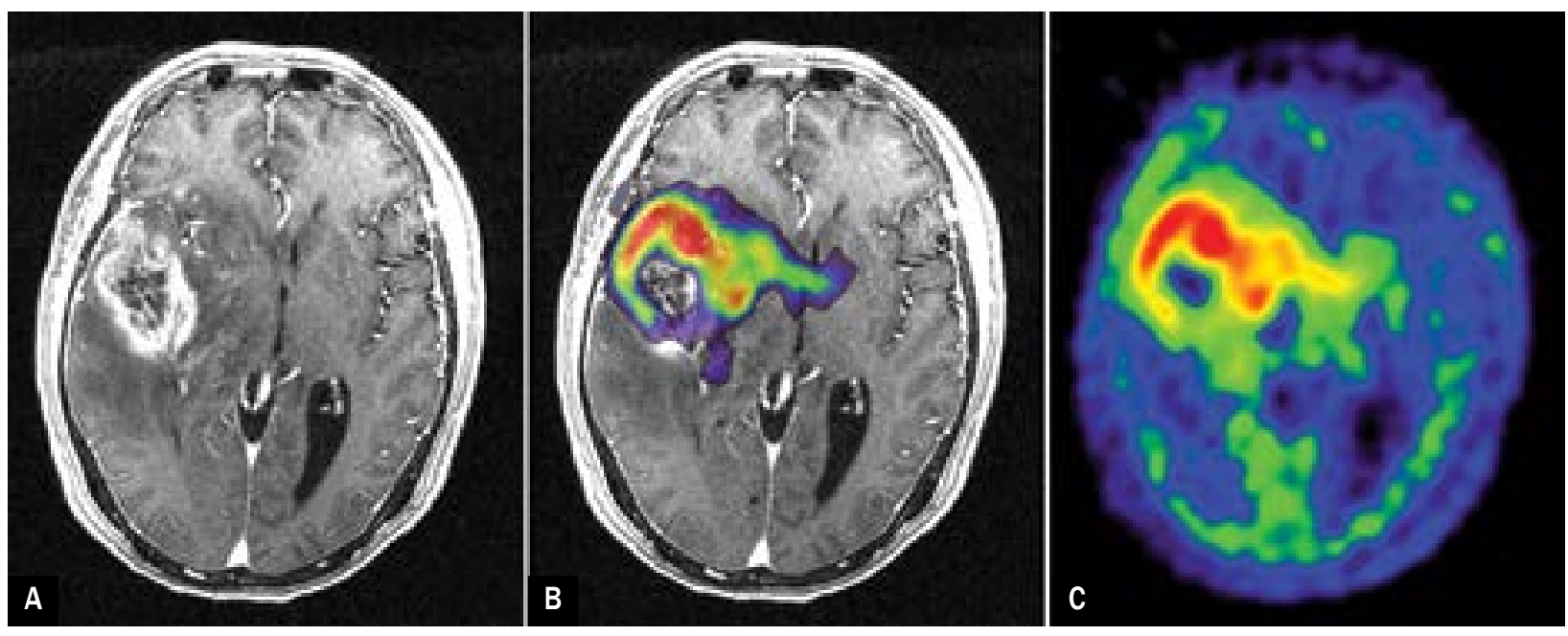

Figure 1. Visual comparison of enhanced MRI with MET PET of primary GBM. A: Tumour contours (green line) in Gd-enhanced T1 MRI. B: Tumour contours comparison in the overlap of MET (TNR $=1.3$ ) and corresponding MRI demonstrated that MET-defined tumour volume extended along commissural fibres of fornix to the contralateral hemisphere. C: General MET PET image of GBM 

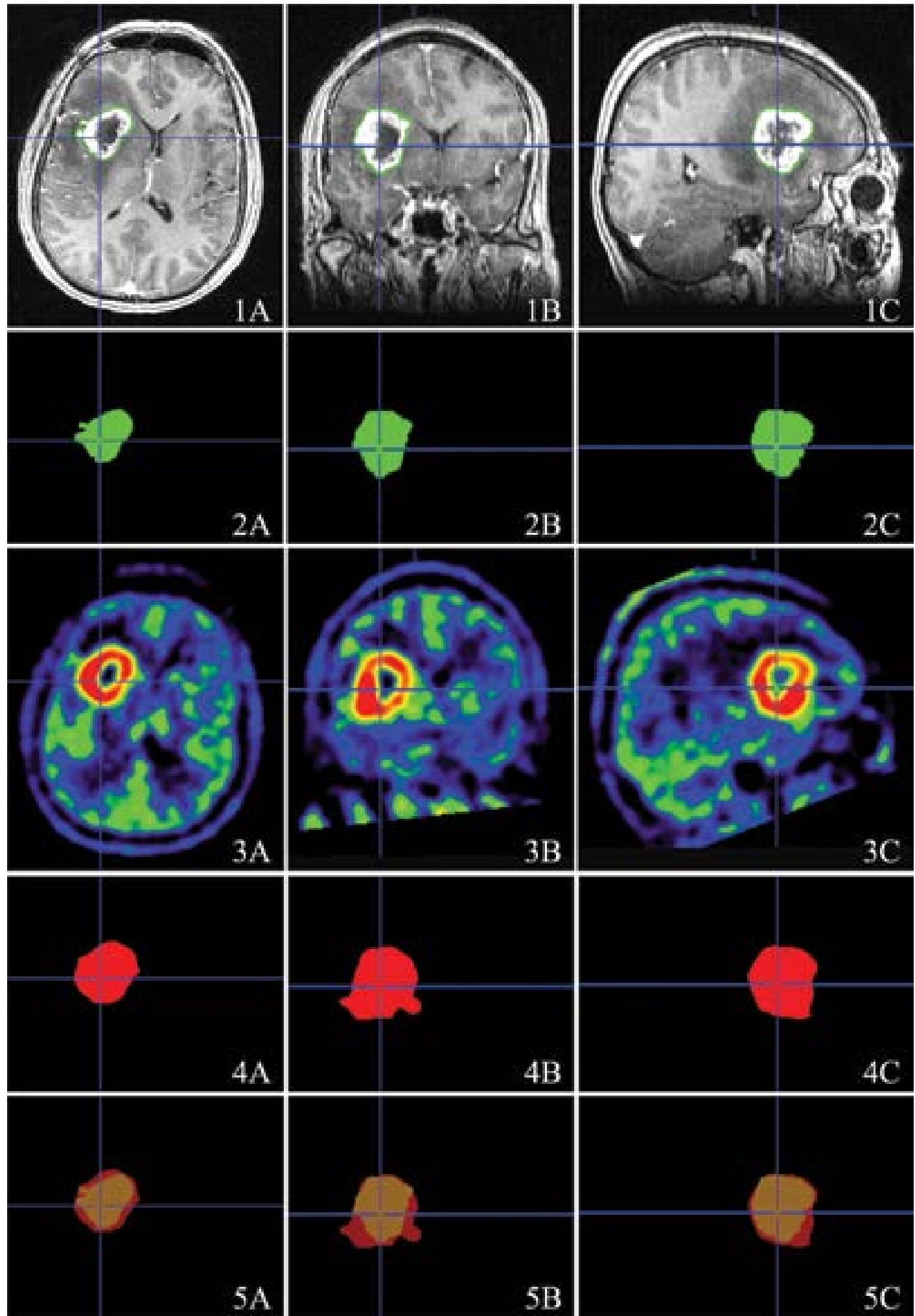

Figure 2. Volumetric analysis of enhanced MRI and MET PET in primary GBM (case 1). Letters A, B and C represent axial, coronal and sagittal views of the same origin, respectively. Row 1: tumour contour (green line) delineated manually in each slice of Gd-enhanced T1 MRI images. Row 2: tumour volume calculated by MRIcron (VMRI is $53.81 \mathrm{~cm}^{3}$ ). Row 3: MET PET images minimally processed to show MET high uptake of GBM. Row 4: Automatically defined metabolic tumour volume (VMET) calculated as $93.31 \mathrm{~cm}^{3}$ (setting threshold: TNR $=1.3$ ). Row 5: tumour volume comparisons between MET PET and the corresponding MRI, dis-MET (volumes contained in MET but not in MRI) is $48.4 \%$ 
corresponding MET PET images are to be expected. PET metabolic imaging using radiolabelled amino acids, such as MET, has been successfully combined with MRI to produce a powerful and reliable technique for defining GBM target volumes in neuronavigation surgery and radiotherapy [17].

To examine the distribution of metabolic abnormalities associated with glioblastoma multiforme relative to MRI, Miwa et al. superimposed contemporaneous MRI on corresponding MET-PET images in 10 patients with newly diagnosed GBM prior to treatment. They found that the MET volume included and exceeded the entire Gd-enhanced volume and that $99.8 \%$ of the MET-enhanced area was located within $30 \mathrm{~mm}$ surrounding of the Gd-enhanced area [7], although the MET volume was assessed by qualitative visual analysis. Galldiks et al. investigated the relationship between MET and Gd-enhanced MRI through volumetric calculation. They reported that the metabolically active tumour volume may be substantially underestimated by Gd-enhanced volume, and they also showed a positive correlation between MET uptake and the volumes of Gd-enhanced area [19]. However, the reference ROI in that study was a two-dimensional circular area that contained limited volumetric information. Moreover, all 12 patients were recurrent GBM. Qualitative, semiquantitative and quantitative methods have all been applied to analyse discrepancies and coincidences between MET PET and MRI; these methods have illustrated the importance of including those areas in the treatment plan $[7,19]$. To date, there has been no precise 3D volumetric study focused on primary GBM.

The aim of the present study was to investigate discrepancies of tumour volumes in primary GBM based on precise volumetric analysis of two imaging modalities. In particular, we investigated tumour volumes metabolically estimated using MET PET that extended regions of Gd-enhanced area. To improve precision and recall, we set strict inclusion criteria. Due to the high proliferation rate of tumour cells, we limited the interval between the MRI and the PET examinations to seven days; this minimised discrepancies between the two image modalities due to tumour growth. This interval was significantly shorter than those used in previous studies $[18,19]$. Furthermore, to obtain representative reference VOI and to segment $V_{\text {MET }}$, we selected three spherical regions with a $10 \mathrm{~mm}$ radius in the unaffected contralateral frontal, occipital and temporal parenchyma. Compared to image processing software in previous studies [19], MRIcron is convenient to acquire and simple to operate. These properties make it suitable and popular for practical clinical use.

As described above, we found that MET PET contours contained and extended beyond most MRI contours in all five patients; this finding was in accordance with the study of Miwa et al. [7]. However, in our study, volumes of high MET uptake were not limited to within $30 \mathrm{~mm}$ of the Gd-enhanced area. Rather, they extended along commissural fibres to the contralateral cerebral hemisphere. This also explains why GBM may exhibit higher proliferation rates in some regions, resulting in asymmetrical and irregular MET PET images.

In volumetric analyses, approximately $39.0 \%$ (17.4$-64.3 \%)$ of metabolic tumour volume was located beyond the Gd-enhanced area. Javier et al. previously performed volumetric research to analyse the contributions of MRI and MET PET to tumour target volume estimates in both highand low-grade glioma. According to this study, approximately $30.22 \%$ (17.4-64.3\%) of MET-defined tumour volume did not overlap with MRI-defined volumes in GBM [18]. However, it must be noted that seven out of ten (70\%) patients were recurrent GBM. This clinical profile significantly affected the analysis, as the authors included the resection cavity when defining tumour volume. These MET-specific volumes are especially important. Recently, John et al. reported findings that support the interpretation that MET delineates non-contrast enhancing tumour regions at high risk for recurrence [12]. Although we performed no statistical estimation, due to our limited sample size, our results nevertheless indicated larger $\mathrm{V}_{\mathrm{MET}}$ estimates associated with VMRI, in line with a previous study [19].

Concerning the specific results in the volumetric analysis observed in our group, it is relevant to discuss the inclusion of volumes with a lack of MET uptake related to central necrosis in the final volume of MET PET. These areas were included in order to facilitate comparison with MRI, which typically includes these areas defining the final surgical target volume.

\section{Limitations}

Our study was performed in a clinical setting, and therefore may reflect a natural bias. Additionally, exact volumetric calculation and comparison could only be performed on patients with presurgical 3D acquisition of both Gd-enhanced MRI and MET PET. As a consequence, the number of patients limits further statistical calculation, and may constrain our conclusions. Although we selected skilled radiologists to delineate MRI volume, there will have been subjective error due to the manually drawn contours.

\section{Conclusion}

GBM is a diffusely infiltrating and widespread malignant neoplasm that, even at the time of diagnosis, typically invades multiple lobes and both hemispheres of the brain. Metabolic tumour volume is substantially underestimated by Gd-enhanced area in primary GBM. MET PET contours contain and extend beyond most MRI contours. In our experience, approximately $40 \%$ of MET high uptake volumes are not included in MRI tumour volumes. Quantitative volumetric information derived from MET uptake is helpful in defining tumour targets and designing individualised therapy strategies for primary GBM. 


\section{References}

1. Komori T, Sasaki H, Yoshida K. [Revised WHO Classification of Tumours of the Central Nervous System:Summary of the Revision and Perspective]. No Shinkei Geka. 2016; 44(8): 625-635, doi: 10.11477/ mf.1436203347, indexed in Pubmed: 27506840.

2. Faguer $\mathrm{R}$, Tanguy $\mathrm{JY}$, Rousseau A, et al. Early presentation of primary glioblastoma. Neurochirurgie. 2014; 60(4): 188-193, doi: 10.1016/j. neuchi.2014.02.008.

3. Stupp R, Tonn JC, Brada M, et al. High-grade malignant glioma: ESMO Clinical Practice Guidelines for diagnosis, treatment and follow-up. Annals of Oncology. 2010; 21(Supplement 5): v190-v193, doi: 10.1093/annonc/mdq187.

4. Grosu AL, Weber WA, Riedel E, et al. L-(methyl-11C) methionine positron emission tomography for target delineation in resected high-grade gliomas before radiotherapy. Int J Radiat Oncol Biol Phys. 2005; 63(1): 64-74, doi: 10.1016/j.ijrobp.2005.01.045, indexed in Pubmed: 16111573.

5. Kelly P, Daumas-Duport C, Kispert D, et al. Imaging-based stereotaxic serial biopsies in untreated intracranial glial neoplasms. Journal of Neurosurgery. 1987; 66(6): 865-874, doi: 10.3171/ jns.1987.66.6.0865.

6. Watanabe M, Tanaka R, Takeda N. Magnetic resonance imaging and histopathology of cerebral gliomas. Neuroradiology. 1992; 34(6): 463-469, doi: 10.1007/bf00598951.

7. Miwa K, Shinoda J, Yano H, et al. Discrepancy between lesion distributions on methionine PET and MR images in patients with glioblastoma multiforme: insight from a PET and MR fusion image study. J Neurol Neurosurg Psychiatry. 2004; 75(10): 1457-1462, doi: 10.1136/ jnnp.2003.028480, indexed in Pubmed: 15377696.

8. Jacobs AH, Li H, Winkeler A, et al. PET-based molecular imaging in neuroscience. European Journal of Nuclear Medicine and Molecular Imaging. 2003; 30(7): 1051-1065, doi: 10.1007/s00259-003-1202-5.

9. Ogawa T, Shishido F, Kanno I, et al. Cerebral glioma: evaluation with methionine PET. Radiology. 1993; 186(1): 45-53, doi: 10.1148/ radiology.186.1.8380108, indexed in Pubmed: 8380108.

10. Okita Y, Kinoshita M, Goto T, et al. 11C-methionine uptake correlates with tumor cell density rather than with microvessel density in glioma: A stereotactic image-histology comparison. Neurolmage. 2010; 49(4): 2977-2982, doi: 10.1016/j.neuroimage.2009.11.024.

11. Jiang H, Cui Y, Liu X, et al. Patient-Specific Resection Strategy of Glioblastoma Multiforme: Choice Based on a Preoperative Scoring Scale. Annals of Surgical Oncology. 2017; 24(7): 2006-2014, doi: 10.1245/ s10434-017-5843-1.

12. Lucas J, Serrano N, Kim H, et al. 11C-Methionine positron emission tomography delineates non-contrast enhancing tumor regions at high risk for recurrence in pediatric high-grade glioma. Journal of Neuro-Oncology. 2017; 132(1): 163-170, doi: 10.1007/s11060-016-2354-z.

13. Susheela SP, Revannasiddaiah S, Madhusudhan N, et al. The demonstration of extension of high-grade glioma beyond magnetic resonance imaging defined edema by the use of (11) C-methionine positron emission tomography. J Cancer Res Ther. 2013; 9(4): 715-717, doi: 10.4103/0973-1482.126464, indexed in Pubmed: 24518724.

14. Pirotte B, Levivier M, Goldman S, et al. POSITRON EMISSION TOMOGRAPHY-GUIDED VOLUMETRIC RESECTION OF SUPRATENTORIAL HIGH-GRADE GLIOMAS. Neurosurgery. 2009; 64(3): 471-481, doi: 10.1227/01.neu.0000338949.94496.85.

15. Yoo M, Paeng J, Cheon G, et al. Prognostic Value of Metabolic Tumor Volume on 11C-Methionine PET in Predicting Progression-Free Survival in High-Grade Glioma. Nuclear Medicine and Molecular Imaging. 2015; 49(4): 291-297, doi: 10.1007/s13139-015-0362-0.

16. Tanaka Y, Nariai T, Momose T, et al. Glioma surgery using a multimodal navigation system with integrated metabolic images. Journal of Neurosurgery. 2009; 110(1): 163-172, doi: 10.3171/2008.4.17569.

17. Pirotte B, Goldman S, Dewitte 0 , et al. Integrated positron emission tomography and magnetic resonance imaging-guided resection of brain tumors: a report of 103 consecutive procedures. Journal of Neurosurgery. 2006; 104(2): 238-253, doi: 10.3171/jns.2006.104.2.238.

18. Arbizu J, Tejada S, Marti-Climent JM, et al. Quantitative volumetric analysis of gliomas with sequential MRI and 11C-methionine PET assessment: patterns of integration in therapy planning. European Journal of Nuclear Medicine and Molecular Imaging. 2012; 39(5): 771-781, doi: 10.1007/s00259-011-2049-9.

19. Galldiks N, Ullrich R, Schroeter M, et al. Volumetry of [(11)C]-methionine PET uptake and MRI contrast enhancement in patients with recurrent glioblastoma multiforme. Eur J Nucl Med Mol Imaging. 2010; 37(1): 84-92, doi: 10.1007/s00259-009-1219-5, indexed in Pubmed: 19662410.

20. Kracht LW, Miletic H, Busch S, et al. Delineation of brain tumor extent with [11C]L-methionine positron emission tomography: local comparison with stereotactic histopathology. Clin Cancer Res. 2004; 10(21): 7163-7170, doi: 10.1158/1078-0432.CCR-04-0262, indexed in Pubmed: 15534088.

21. Brown T, Brennan M, Li M, et al. Association of the Extent of Resection With Survival in Glioblastoma. JAMA Oncology. 2016; 2(11): 1460, doi: 10.1001/jamaoncol.2016.1373.

22. Kracht L, Friese M, Herholz K, et al. Methyl-[11C]---methionine uptake as measured by positron emission tomography correlates to microvessel density in patients with glioma. European Journal of Nuclear Medicine and Molecular Imaging. 2003; 30(6): 868-873, doi: 10.1007/ s00259-003-1148-7.

23. Kato T, Shinoda J, Nakayama N, et al. Metabolic Assessment of Gliomas Using11C-Methionine, [18F] Fluorodeoxyglucose, and11C-Choline Positron-Emission Tomography. American Journal of Neuroradiology. 2008; 29(6): 1176-1182, doi: 10.3174/ajnr.a1008.

24. Singhal T, Narayanan TK, Jacobs MP, et al. 11C-Methionine PET for Grading and Prognostication in Gliomas: A Comparison Study with 18F-FDG PET and Contrast Enhancement on MRI. Journal of Nuclear Medicine. 2012; 53(11): 1709-1715, doi: 10.2967/jnumed.111.102533. 